\title{
A REMARK ON CONTINUED FRACTIONS
}

\section{H. Davenport}

Very little is known about the continued fraction expansion of algebraic numbers other than quadratic irrationals, though Roth's theorem imposes a limitation on the rapidity with which the partial quotients $a_{n}$ can increase. In particular, it has never been proved that there exists such a number for which the sequence $a_{n}$ is unbounded, though this is almost certainly true.

The following much easier question was put to me by Dr. Schinzel: given $\mathrm{N}$, however large, do there exist algebraic numbers other than quadratic irrationals which have infinitely many partial quotients greater than $\mathrm{N}$ ? The following simple result answers this question, but does so without throwing any light on the deeper problems.

Let $\theta$ be any irrational number, and let $\mathrm{P}$ be any large prime. Then one at least of the numbers

$$
\mathrm{P}^{2} \theta, \theta, \theta+1 / \mathrm{P}, \cdots, \theta+(\mathrm{P}-1) / \mathrm{P}
$$

has $\mathrm{a}_{\mathrm{n}}>\mathbf{P}-2$ for infinitely many $\mathrm{n}$.

To prove this, we consider the convergents $\mathrm{p}_{\mathrm{n}} / \mathrm{q}_{\mathrm{n}}$ to the irrational number $\mathrm{P} \theta$. They satisfy the inequality

$$
\left|\mathbf{p} \theta-\frac{\mathbf{p}_{\mathbf{n}}}{\mathbf{q}_{\mathbf{n}}}\right|<\frac{1}{\mathbf{q}_{\mathrm{n}}^{2}} .
$$

If $q_{n}$ is divisible by $P$ for infinitely many $n$, we put $q_{n}=P q_{n}^{\prime}$ and get infinitely many rational approximations $p_{n} / q_{n}^{1}$ to $P^{2} \theta$ satisfying the inequality

$$
\left|\mathrm{P}^{2} \theta-\frac{\mathrm{p}_{\mathrm{n}}}{\mathrm{q}_{\mathrm{n}}^{\prime}}\right|<\frac{1}{\mathrm{P}\left(\mathrm{q}_{\mathrm{n}}^{\prime}\right)^{2}} .
$$

This implies that infinitely many of the partial quotients in the continued fraction for $\mathbf{P}^{2} \theta$ are greater than $\mathrm{P}-2$. (This is a consequence of well-known properties of continued fractions; see for example Theorems 184 and 163 in Hardy and Wright's Introduction to the theory of numbers.)

In the contrary case, $\mathrm{q}_{\mathrm{n}}$ is relatively prime to $\mathrm{P}$ for all sufficiently large $\mathrm{n}$. Thus we can determine an integer $A_{n}$ such that

$$
\mathrm{p}_{\mathrm{n}} \equiv \mathrm{A}_{\mathrm{n}} \mathrm{q}_{\mathrm{n}}(\bmod \mathrm{P}), \quad 0 \leq \mathrm{A}_{\mathrm{n}}<\mathrm{P} .
$$

One at least of the integers $0,1, \cdots, P-1$ must occur infinitely often as $A_{n}$. If $A$ is such an integer, we can write

$$
p_{n}=A q_{n}+P r_{n}
$$

Received August 3, 1964. 
for the corresponding infinite set of values $n$, where $r_{n}$ is an integer. Now

$$
\left|\theta-\frac{\mathrm{A}}{\mathbf{P}}-\frac{\mathbf{r}_{\mathrm{n}}}{\mathbf{q}_{\mathrm{n}}}\right|<\frac{1}{\mathrm{Pq}_{\mathrm{n}}^{2}}
$$

for infinitely many $\mathrm{n}$, and again this implies that $\theta-\mathrm{A} / \mathrm{P}$ has infinitely many partial quotients greater than $P-2$. This proves the result.

Ohio State University

and

Trinity College

Cambridge, England 